\title{
Correlation Analysis of Th17/Treg Cells and PTPN22 Gene Polymorphism in HT Patients with Different lodine Nutritional Status
}

\author{
Wang Cuicui ${ }^{1}$, Mu Zhaoxin ${ }^{2}$, Hou Zhenjiang ${ }^{2,}{ }^{,}$, Chen Yunxia ${ }^{3}$, Liu Jianfeng ${ }^{3}$, Ma Jinqun ${ }^{3}$, \\ Liu Chunyan ${ }^{3}$ \\ ${ }^{1}$ Department of Medical Technology of Cangzhou Medical College, Cangzhou, China \\ ${ }^{2}$ Institute of Thyroid Diseases Affiliated to Cangzhou Medical College, Cangzhou Thyroid Disease Engineering Technology Research Center, \\ Cangzhou, China \\ ${ }^{3}$ Cangzhou People's Hospital Endocrinology, Cangzhou, China
}

Email address:

houzhenjiang@sina.com (Hou Zhenjiang)

${ }^{*}$ Corresponding author

\section{To cite this article:}

Wang Cuicui, Mu Zhaoxin, Hou Zhenjiang, Chen Yunxia, Liu Jianfeng, Ma Jinqun, Liu Chunyan. Correlation Analysis of Th17/Treg Cells and PTPN22 Gene Polymorphism in HT Patients with Different Iodine Nutritional Status. Science Journal of Public Health.

Vol. 9, No. 2, 2021, pp. 64-71. doi: 10.11648/j.sjph.20210902.15

Received: March 8, 2021; Accepted: April 19, 2021; Published: April 26, 2021

\begin{abstract}
Background: The incidence of Hashimoto's thyroiditis (HT) may be related to environmental, immune, and genetic factors. Different iodine nutritional status can affect thyroid function. Th17/Treg cell imbalance is involved in the pathogenesis of autoimmune thyroid diseases, and PTPN22 is an important susceptibility gene related to autoimmune diseases. To study the relationship between Th17/Treg cells and cytokines and PTPN22 gene polymorphism in HT patients with different iodine nutritional status is to determine the role of immune and genetic factors in the pathogenesis of HT. Objective: To investigate the correlation between Th17/Treg cells and factors protein tyrosine phosphatase non-receptor type 22 (PTPN22) gene-1123G $>\mathrm{C}$ site (rs2488457) polymorphism in peripheral blood mononuclear cells (PBMC) of Hashimoto's thyroiditis (HT) patients with different iodine nutritional status. Methods: $100 \mathrm{HT}$ patients and 60 healthy subjects in Cang Zhou are selected from 2019 to 2020. Flow cytometry and real-time fluorescent quantitative PCR are used to detecting the proportion and ratio of Th17 and Treg cells in PBMC, as well as retinoic acid-related orphan receptor- $\gamma \mathrm{t}(\mathrm{ROR}-\gamma \mathrm{t})$ and fork head/winged spiral transcription factor 3 (Foxp3) mRNA expression levels. Single allele-specific primer-polymerase chain reaction (SASP-PCR) technology is taken to sequence PTPN gene promoter-1123SNP leading to calculate the gene Type and allele frequency. Results: Th17 and Treg cells and factors in the PBMC of HT patients have a certain correlation with the-1123G $>$ C locus of PTPN22 gene (rs2488457). The distribution frequency of rs2488457 genotype is consistent with Hardy Weinberg's law of genetic equilibrium. The distribution frequencies of various genotypes and alleles are significantly different between HT patients and the control group $(\mathrm{P}<0.05)$. PTPN22 gene-1123G $>\mathrm{C}$ site $\mathrm{C} / \mathrm{G}, \mathrm{C} / \mathrm{C}$ genotype can increase of the risk of HT, while $\mathrm{G} / \mathrm{G}$ genotype can decrease of the risk of HT. In Conclusion: The polymorphism of rs 2488457 is related to susceptibility to HT. Carrying C/G and C/C genotypes may be risk factors for HT, and G/G genotypes may be protective factors for HT, but it has nothing to do with iodine nutrition status.
\end{abstract}

Keywords: Iodine Nutritional Status, Hashimoto's Thyroiditis, Th17/Treg Cells and Factors, PTPN22, Gene Polymorphism

\section{Introduction}

Autoimmune thyroid disease (AITD) is a clinically common organ-specific autoimmune disease. Its incidence has gradually increased in recent years. Hashimoto's thyroiditis (HT) and
Graves' disease (GD) are the most common, respectively. It is characterized by hypothyroidism and hyperthyroidism with lymphocyte infiltration and autoantibody positive, which is the result of the combined effect of humoral immunity and cellular immunity. HT is the most common organ-specific AITD, accounting for about $22.5 \%$ of thyroid diseases [1]. The 
prevalence of HT in foreign countries is $1 \%$ to $2 \%$, among which men are $0.08 \%$ and women are $0.35 \%$. It is more common in middle-aged women between 20 and 50 years old, and men are younger than women [2]. The prevalence of HT in China accounts for about $7.3 \%$ to $20.5 \%$ of thyroid diseases, and the male to female ratio is 1:6 to $1: 10$, which is more common in women between 30 and 50 years old [1]. The onset of HT may be affected by many factors such as environment, genetics and immunity. In recent years, research on the correlation between HT and Th17/Treg cells and their factors had been carried out. It is believed that Th17/Treg cells and their factors are involved in the pathogenesis of HT and other AITD. Research have found that Th17 cells, not Th1 cells, play a key role in the pathogenesis of HT [3], and the proportion of Th17 cells in PBMC has increased [4]. The proportion of Th17 cells in the PBMC of AITD patients increases significantly, and Hashimoto's hypothyroidism is more obvious, which suggests that the increase in the proportion of Th17 cells may be more closely related to Hashimoto's hypothyroidism [5]. Glick et al. find that there is no significant difference between TregCD4 $4^{+} \mathrm{T}$ cells and the control group, but the inhibitory ability decreases, which suggested that the function of Treg cells in AITD patients decreases [6]. Marazuela et al. reported that the proportion of Treg cells in thyroid tissue and PBMC in AITD patients is significantly higher than that in the control group, and there are functional defects, and its inhibitory ability significantly decreases, and it could not effectively down-regulate the body's immune response to prevent the occurrence of AITD [7]. A large number of basic and clinical research data show that the etiology of AITD is complex, and its pathogenesis is not only related to immune factors, but also closely related to heredity. The protein tyrosine phosphatase nonreceptor 22 (PTPN22) is currently considered to be an important susceptibility gene that related to autoimmune diseases [1], and its abnormal function can lead to rheumatoid arthritis [8], systemic lupus erythematosus [9], myasthenia gravis [10] and other autoimmune related diseases [11]. There have been many reports that the single nucleotide polymorphism (SNP) of PTPN22 is related to the above-mentioned diseases. AITD has similar genetic and immunological basis, but the specific mechanism of action has not been fully elucidated. Therefore, in-depth research on cellular immunity and genetic genetics is helpful to the diagnosis, treatment and prevention of AITD.

\section{Materials and Methods}

\subsection{Research Subjects and Groupings}

100 cases of untreated inpatients with HT who are in the endocrinology department of Cangzhou People's Hospital from September 2019 to August 2020 are selected as the experimental group, including 26 males and 74 females, with an average age of (47.4 \pm 16.8$)$ years. According to the results of thyroid function tests, HT patients are divided into normal thyroid function group (HT-A group, 31 cases), subclinical hypothyroidism group (HT-B group, 46 cases) and clinical hypothyroidism group (HT-C group, 23 cases). A total of 60 health checkups matched by gender and age are selected as the control group (NC group), including 16 males and 44 females, with an average age of $(45.8 \pm 17.6)$ years. There are no statistical differences in gender and age between the groups. All patients meet the diagnostic criteria of HT in the "Guidelines for the Diagnosis and Treatment of Thyroid Diseases in China" formulated by the Endocrinology Branch of the Chinese Medical Association in 2008 [12], and none of them receive relevant treatment. HT inclusion criteria: (1) Abnormal serum thyroid-stimulating hormone levels, and anti-thyroid peroxidase antibodies and/or anti-thyroglobulin antibodies are beyond the upper limit of the normal range; (2) The thyroid is hard, with or without goiter; (3) Confirmed by puncture cytology or pathological examination after surgery. HT exclusion criteria: (1) recent systemic lupus erythematosus, rheumatoid arthritis, asthma, pernicious anemia, multiple sclerosis, systemic sclerosis, etc.; (2) recent ( $\leq 6$ months) viral or bacterial infection Medical history; (3) Recent ( $\leq 6$ months) pregnancy or history of childbirth; (4) History of malignant tumors. The assessment of iodine nutritional status adopts the standards revised by the World Health Organization (WHO), the United Nations Children's Fund (UNICEF) and the International Council for the Control of Iodine Deficiency Disorders (ICCIDD) in 2007: Median urinary iodine $(\mathrm{MUI})<100 \mu \mathrm{g} / \mathrm{L}$ is iodine deficiency, $100 \sim 199 \mu \mathrm{g} / \mathrm{L}$ is the right amount of iodine, $200 \sim$ $300 \mu \mathrm{g} / \mathrm{L}$ is iodine excess, and $300 \mu \mathrm{g} / \mathrm{L}$ or more is iodine excess [13]. This research is approved by the ethics committee, and all the research subjects sign an informed consent form.

\subsection{Research Methods and Projects}

\subsubsection{Specimen Collection and Processing}

(1) Specimen collection: All subjects are fasted for more than 12 hours, so as to collect $10-20 \mathrm{ml}$ of fasting midsection urine that is placed in a clean polyethylene plastic tube, and collect fasting heparin for anticoagulation at 8:00 10:00 $5 \mathrm{~mL}$ of venous blood that is stored in a refrigerator. (2) Peripheral blood mononuclear cell (PBMC) separation: Separated PBMC by Ficoll density gradient centrifugation, adjusting the concentration density of PBMC of each sample to $1 \times 10^{6} / \mathrm{L}$, and suspending it in the incubation solution of RPMI640 fetal bovine serum. Putting the PBMC suspension in a 24 -well culture plate, add $25 \mu \mathrm{g} / \mathrm{L}$ of phorbol ester (Alexis, USA) and $1 \mathrm{mg} / \mathrm{L}$ of inomycin (Alexis, USA) to each well, and incubate in a $37^{\circ} \mathrm{C}$ incubator for 4 hours. Collecting cells for later use. (3) RNA extraction and cDNA synthesis: Trizol method is used to extract total RNA from PBMCs of HT patients and control group, formaldehyde denaturing electrophoresis and absorbance value (A260/A280) to identify RNA quality. Taking the RNA with the ratio of A260/A280 above 1.9 and at least 2 bands in electrophoresis for the following experiment, and adjusting the RNA concentration of each sample to $0.5 \mu \mathrm{g} / \mathrm{L}$ for use.

\subsubsection{Project Testing}

(1) Urinary iodine determination: Using the principle of iodine-catalyzed arsenic-cerium reaction, the urinary iodine content is detected by arsenic-cerium catalytic spectrophotometry (WS/T107-2006). The kit is produced by 
Wuhan Zhongsheng Biochemical Technology Co. Ltd., and the instrument used Hitachi 7600-110 type automatic biochemical analyzer. (2) Detection of the ratio of Th17 and Treg cells: Th17 cells are labeled with a human Th17 cell detection kit (FITC/CD3, PE/CD4, Alexa Fluor647/IL-17, Biolegend, USA), and a human Treg cell detection kit (FOXP3 Alexa Fluor 488/CD4 PE-Cy5/CD25PE) to label Treg cells, use FACSCalibur flow cytometer (BD company, USA), according to the instructions, adding the corresponding fluorescent dye-labeled antibody into the flow tube containing $100 \mu \mathrm{L}$ of blood sample to incubate at room temperature for 15 minutes in the dark, adding red blood cell lysate, mix and incubate at $4^{\circ} \mathrm{Cfor} 10$ minutes in the dark, and adding PBS solution and mix thoroughly and test on the machine. The test results are based on the percentage from Cell Quest software. (3) Polymerase chain reaction (PCR) amplification and genotyping: Trizol method is used to extract total RNA from the sample. cDNA is synthesized according to the reverse transcription procedure. DNA is extracted, and single allele-specific primers-polymerase chain reaction (PCR-SASP) technology to amplify the $1123 \mathrm{G} / \mathrm{C}$ site upstream of the PTPN gene promoter. Design 3 primers with reference to the DNA sequence of PTPN22 in Gen Bank, including 1 common upstream primer $(\mathrm{R})$ and 2 downstream primers (F1 and F2) for specific alleles at the 3 'end, upstream primer sequence R: 5'-ACCCTGCAT ATGT AATGCTGGT-3', downstream primer sequence $\mathrm{F} 1$ : 5'-CATTGAGAGGTTATGCAAGCTC-3' and downstream primer sequence F2: CATTGAGAGGTTATGCAA-GCTG-3', the primer is synthesized by Shanghai Jierui Biological Engineering Co. Ltd. Each specimen is amplified using F1 and F2. F1 and R formed a pair of primers to amplify the G allele, and $\mathrm{F} 2$ and $\mathrm{R}$ formed a pair of primers to amplify the $\mathrm{C}$ allele. The genotype is determined according to the presence or absence of PCR products. Using $0.2 \mu \mathrm{g}$ DNA as a template, PCR amplification is carried out in a reaction volume of $30 \mu \mathrm{L}$. The reaction conditions are: denaturation at $95^{\circ} \mathrm{C}$ for $5 \mathrm{~min}$, denaturation at $95^{\circ} \mathrm{C}$ for $40 \mathrm{~s}$, annealing at $60^{\circ} \mathrm{C}$ for $40 \mathrm{~s}$, extension at $72^{\circ} \mathrm{C}$ for $40 \mathrm{~S}$, and extension at $72^{\circ} \mathrm{C}$ for $7 \mathrm{~min}$. After storage at $4^{\circ} \mathrm{C}$, after 40 cycles, the amplified products are electrophoresed on a $2 \%$ agarose gel, and genotype identification is performed after staining. The PCR amplification product is purified and sequenced by the primer synthesis company. According to the sequencing file, the peak map of the target site is analyzed to determine the genotype of the site.

\subsubsection{Statistical Methods}

Using SPSS 20.0 statistical software for statistical analysis.
Each group of genotypes and allele frequencies are tested for Hardy-Weinberg genetic balance to confirm whether they comply with the balance law, and to calculate the genotype frequencies and allele frequencies of each polymorphic locus. The $\chi^{2}$ test is used for comparison, and the odds ratio (OR) is used to expressing the relative risk, and $\mathrm{P}<0.05$ is considered as the difference is statistically significant.

\section{Results}

\subsection{SASP-PCR Analysis of PTPN22 Gene at 1123 Locus}

The length of the amplified fragment of the PTPN22 promoter is $268 \mathrm{bp}$. The primer F1 is for allele G (wild type), and the primer F2 is for allele $\mathrm{C}$ (mutant type). When the genotype is GG, only the downstream primer F1 can amplify the PCR product; when the genotype is CC, only the downstream primer F2 can amplify the PCR product; when the genotype is GC, both downstream primers F1 and F2 can be Amplify the PCR product. The results of this study detect three genotypes at the $1123 \mathrm{G}>\mathrm{C}$ locus (rs2488457) of the PTPN22 gene, which are $\mathrm{G} / \mathrm{G}, \mathrm{C} / \mathrm{G}$, and $\mathrm{C} / \mathrm{C}$ genotypes, as shown in Figure 1.

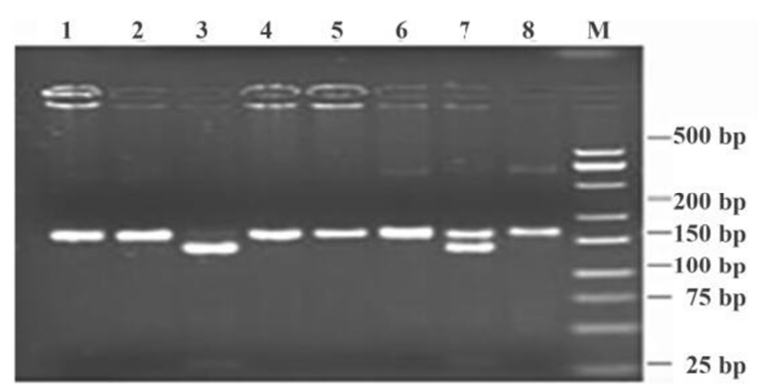

Figure 1. Results of PCR amplification with sequence specificprimers at 1 123 site in promoter region.

Note: Marker: 3. CC homozygote, 7. GG heterozygote, others are CG homozygote.

\subsection{Comparison of the Genotypes and Allele Frequencies of the PN22 Gene Qigongzi-1123 Polymorphism in HT Patients and the Control Group}

The genotype and allele frequency distribution of PTPN22 gene at $1123 \mathrm{G}>\mathrm{C}$ locus (rs2488457) in HT and control group conformed to Hardy Weinberg's law of genetic balance, and the difference between the two groups is statistically significant $(\mathrm{P}<0.05)$. Table 1 .

Table 1. Comparison of genotypes and allele frequencies between HT patients and control groups [ $\mathrm{n}(\%)]$.

\begin{tabular}{lllll}
\hline & HT Group & Control Group & $\boldsymbol{\chi 2}^{\mathbf{2}}$ & P \\
\hline genotype & & & & \\
G/G & $28(28 \%)$ & $21(35 \%)$ & 5.672 & \\
C/G & $50(50 \%)$ & $22(36.7 \%)$ & & \\
C/C & $22(22 \%)$ & $17(28.3 \%)$ & & 0.046 \\
Allele frequency & & & 5.126 & \\
G & $86(53.8 \%)$ & $83(51.9 \%)$ & & \\
C & $74(46.2 \%)$ & $77(48.1 \%)$ & & \\
\hline
\end{tabular}




\subsection{The Relative Risk of Rs2488457 Genotype and Alleles to HT}

According to the generalized genetic model, calculating the corresponding ratio $(\mathrm{OR})$ value and $\mathrm{P}$ value of the genotype and allele of PTPN22 gene at rs2488457 locus. OR value represents relative risk, and the theoretical calculation formula of OR value is $\mathrm{OR}=(\mathrm{a} / \mathrm{b}) /(\mathrm{c} / \mathrm{d})$. The difference has statistically significant when $\mathrm{P}<0.05$. The results show that the $\mathrm{C} / \mathrm{G}$ and $\mathrm{C} / \mathrm{C}$ genotypes at $1123 \mathrm{G}>\mathrm{C}$ of PTPN22 gene can significantly increase the risk of $\mathrm{HT}$, and carrying $\mathrm{G} / \mathrm{G}$ genotype can reduce the risk of HT. Carrying $\mathrm{C} / \mathrm{G}$ and $\mathrm{C} / \mathrm{C}$ genotypes may be risk factors for the occurrence of HT (OR values are 1.727 and 1.628 , respectively), and carrying $\mathrm{G} / \mathrm{G}$ genotypes may be protective factors for the occurrence of HT (OR values are 0.722 ), see Table 2 .

Table 2. Relative risk of rs2488457 genotype and alleles to HT.

\begin{tabular}{lccccc}
\hline & \multirow{2}{*}{ OR } & \multicolumn{2}{c}{$\mathbf{9 5 \% C I}$} & \multirow{2}{*}{$\boldsymbol{2}$} & \multirow{2}{*}{$\mathbf{P}$} \\
\cline { 3 - 4 } & & lower & upper & & \\
\hline genotype & & & & & \\
$\mathrm{G} / \mathrm{G}$ & 0.722 & 0.128 & 0.261 & 13.815 & 0.054 \\
$\mathrm{C} / \mathrm{G}$ & 1.727 & 0.203 & 1.014 & 10.694 & 0.041 \\
$\mathrm{C} / \mathrm{C}$ & 1.628 & 0.136 & 1.283 & 12.316 & 0.049 \\
Allele frequency & & & & \\
$\mathrm{G}$ & 1.078 & -0.348 & 0.437 & 1.513 & 0.437 \\
$\mathrm{C}$ & 0.928 & -0.428 & 0.528 & 1.326 & 0.528 \\
\hline
\end{tabular}

\section{Discussion}

Most research believe that the onset of AITD is the result of the combined effects of environment, genetics, and immune imbalance, among which immune factors are recognized as the main cause of disease. Th17/Treg is a pair of Th cell subgroups with pro-inflammatory/anti-inflammatory effects, with independent differentiation and regulation mechanisms, and mutual inhibition, which has been proven to play an important role in the pathogenesis of AITD. Th17 cells secrete high levels of IL-17, so that pro-inflammatory factors induce the production of a variety of pro-inflammatory cytokines and chemokines, and then participate in the body's inflammatory response and immune defense [14, 15]. Treg cells prevent the activation of self-reactive $T$ cells by secreting cytokines, direct contact, etc. and exert immunosuppressive effects. They can also regulate the activation of effector $T$ cells at the transcriptional level through Foxp3, thereby inhibiting the occurrence of AID. Th17/Treg cell balance can maintain the body in a steady state of immune tolerance. In inflammatory reactions or autoimmune diseases, the differentiation of $\mathrm{T}$ cells is affected. Th17 cell differentiation is enhanced, and Treg cell differentiation is inhibited, which disrupts the Th17/Treg cell balance, resulting in immune damage to the body [16]. The author's previous research results indicate that thyroid function changes in HT patients with different iodine nutritional status, often accompanied by significant increases in TPOAb and TgAb titers, and are related to Th17/Treg cells and their cytokines and transcription factors. It further confirms that HT is mainly mediated by Th17 cells and is accompanied by the production of autoantibodies, indicating that while HT patients have abnormal humoral immunity, cellular immunity also plays an important role. The PTPN22 gene is located in the short arm of human chromosome 1 (1p13.3-p13.1), and it encodes a lymphoid protein tyrosine phosphatase (Lyp) consisting of 807 amino acid residues. The PTPN22 gene is mononuclear nucleotide polymer-Phism, SNPs) mainly including $1858 \mathrm{C}>\mathrm{T}, 1123 \mathrm{G}>\mathrm{C}, 788 \mathrm{G}>\mathrm{A}$, etc. The protein expressed after its mutation weakens or disappears to inhibit the immune response and triggers AID. $1858 \mathrm{C}>\mathrm{T}$ The structural variation of Lyp $\mathrm{P} 1$ region affects the binding of Lyp SH3 region and Csk kinase, and the half-life of Lyp is shortened, which together promote the overactivation of T cells [17]. 1123G $>\mathrm{C}$ can affect the binding site of central activated protein 4 , and may affect the transcription efficiency of Lyp [18]. 788G $>$ A can reduce the basic physical activity of Lyp, which is manifested as the loss of T cell function [19]. Therefore, PTPN22 gene SNPs may have a protective effect in AID [7]. PTPN22 gene plays a negative regulatory role in the $\mathrm{T}$ cell signaling pathway. The first recognized polymorphism of PTPN22 gene is $\mathrm{C} / \mathrm{T}$ at position 1858 of codon 620 , which cause the mutation of arginine to tryptophan. When the 620th arginine (Arg) of PTPN22 is mutated to tryptophan (Try), the activity of PTPN22 is enhanced, which can inhibit the signal transduction of TCR, thereby promoting the occurrence of autoimmunity. Lyp has a negative regulatory effect on the activation of T cells [20]. If the T gene at 1123 is mutated, its activation degree will be enhanced, which will interfere with the body's immune tolerance, thereby inducing AID and increasing the risk of autoimmune thrombocytopenia. The $\mathrm{C} \rightarrow \mathrm{T}$ mutation at position 1858 of the PTPN22 gene causes the TGG codon coding for tryptophan (Trp) to replace the codon CGG coding for arginine (Arg). The structural change leads to functional defects, thereby potentially changing LYP as $\mathrm{T}$ The normal function of negative regulators of cell activity makes $\mathrm{T}$ cell signal transduction obstacles, leading to the occurrence of AID [21]. It is currently considered to be an important susceptibility gene related to AID.

PTPN22 is involved in the occurrence of cellular immunity and inflammation, and is related to a variety of AIDs [22]. Research has found that PTPN22 is a susceptibility gene associated with multiple AIDs. In 2004, Bottini et al. first reported that PTPN22 C1858T in two independent populations in North America and Sardinia, Italy was related to the onset of type 1 diabetes (T1DM). In 2006, Kawasaki et al find that after a full genetic scan of T1DM patients, the Japanese of PTPN22 has 5 SNP sites [23]. Among them, the $1123 \mathrm{G}>\mathrm{C}$ mutation is a mutation site that has a higher frequency of autoimmune diseases and is closely related to T1DM. It is found in both Korean and Caucasian populations. In 2004, Begovich et al. find for the first time that the PTPN22 $\mathrm{C} 1858 \mathrm{~T}$ allele exists in RA patients, and it is related to rheumatoid factor (RF)-positive RA, but has nothing to do with RF-negative RA. PTPN22 C1858T in the European and North American white populations is related to RA and may be one of the susceptibility genes of RA. Salama [24] finds 
that the severity and activity of disease in RA patients with CT and TT genotypes are significantly higher than that of CC genotypes. However, in Belarus and Greece in Europe, Colombia in South America, and Japan and Turkey in Asia, there is no conclusion that PTPN22 SPN is related to RA. The PTPN22 C1858T allele is not detected in 776 cases of RA and the Han people in the control group in Guangdong [25], Guizhou [26] and Shandong [27] in China, further suggesting that the PTPN22 $1858 \mathrm{C} / \mathrm{T}$ polymorphism is not related to the incidence of RA in the Han people. Most research results [28] indicate that the PTPN22 C1858T polymorphism is related to the pathogenesis of SLE, but there are also reports that the PTPN22 C1858T polymorphism in white populations such as North America, the United Kingdom and Finland has nothing to do with simple SIE. Hamza et al. [29] find that there is no statistically significant difference in the $\mathrm{T}$ allele frequency between Egyptian children and adolescents with SLE and the control group, but the difference between R620W and SLE patients with thyroid disease and the control group is statistically significant $(\mathrm{P}=0.002)$. Piotrowski et al. conduct related research on 150 Polish SLE patients and reach similar conclusions $(\mathrm{OR}=2.552,95 \% \mathrm{CI}=0.6748 \sim 9.6400, \mathrm{P}=0.1675)$, analyze the $\mathrm{T}$ allele typing, and speculate on environmental factors and heredity Heterogeneity promoting the role of PTPN22 C1858T in the pathogenesis of SLE. Recently, Tang et al. [30] conduct PTPN22 SPN genotype analysis on 358 RA, 713 SLE patients and controls $(564,672)$, and find that the clinical features of RA and SLE are significantly related to SNPs. rs2476601 is found in RA, SLE and SLE patients. The control group lack polymorphism. The PTPN22 genes rs1217414 and rs3811021 may be the common protective factors of RA and SLE susceptibility in Chinese Han people.

The research finds that PTPN22 is related to AITD in European populations, but there are obvious geographic differences in the frequency of $1858 \mathrm{~T}$ alleles in different populations. In healthy European populations, the frequency of $1858 \mathrm{~T}$ alleles shows a decreasing trend from north to south, and the northern Finnish population accounts for $15.6 \%$, The southern Italian and Spanish populations are about $6 \%$, while there are almost no $\mathrm{T}$ alleles in the central African and Asian populations [31]. Alkhateeb et al. [32] report that the frequency of the $\mathrm{T}$ allele in the AITD population in Tunisia, Africa was $1.2 \%$. Ray et al. [33] study the polymorphism of the PFPN22 gene in Indian idiopathic hypoparathyroidism and find that the PTPN22 1858T allele is in the frequency of occurrence in the Asian population is lower, with patients and controls being $4 / 160(2.5 \%)$ and $5 / 386(1.3 \%)$, respectively $(\mathrm{OR}=1.95)$. The above results are similar to the findings of Ban et al. [34] Asian population and Xue [35] et al. on the low frequency characteristics of PTPN22 T1858 locus in Chinese people. Although the PTPN22 1858T allele has a negative result in AITD [34], it also provides new ideas for further research on PTPN22 C1858T. Pan Ruowang et al. [36] report that there are three genotypes in the PTPN22 1123G>C (rs2488457) polymorphism in the Han people in Wenzhou. The GG, GC and CC genotype frequencies in HT patients are 0.207, 0.627 and 0.167 , respectively, and the $\mathrm{G}$ and $\mathrm{C}$ gene frequencies are 0.520 and 0.480 , the control group genotype frequencies are 0.375 , 0.475 , and 0.150 , the $\mathrm{G}$ and $\mathrm{C}$ gene frequencies are 0.613 and 0.388 , respectively. The PTPN22-1123 locus polymorphism distribution frequency is significantly different between HT patients and the control group $(\mathrm{P}<0.05)$, and the frequency of $\mathrm{C}$ gene in HT patients is significantly higher than that in the control group $(\mathrm{P}<0.05)$. Compared with the $\mathrm{GG}$ genotype, both the $\mathrm{GC}$ and $\mathrm{GG}+\mathrm{GC}$ genotypes can significantly increase in the risk of HT. Carriers of the $-1123 \mathrm{C}$ allele are 1.459 times more likely to develop HT than the $\mathrm{G}$ allele (OR=I.459), 95\% CI $1.03 \sim 2.059$ ). HuoYunli [37] et al. reported that PTPN22 gene $1123 \mathrm{G}>\mathrm{C}$ locus (rs2488457) in Zhangjiakou Han population is $30 \%, 36 \%$, and $34 \%$ for $\mathrm{G} / \mathrm{G}, \mathrm{C} / \mathrm{G}$, and $\mathrm{C} / \mathrm{C}$ genotype frequencies, respectively, and 21.5 for HT patients. $50 \%, 28.5 \%$, the control group $\mathrm{C}$ and $\mathrm{G}$ allele frequencies are $48 \%, 52 \%$, HT patients are $46.5 \%, 53.5$, the distribution frequency of each genotype and allele between HT patients and the control group The difference had statistically significant $(\mathrm{P}<0.05)$, and no $\mathrm{T}$ allele is detected, which is consistent with the results of the rare $T$ allele in the Asian population in the NCBI SNP database. The results of this research show that PTPN22 gene $1123 \mathrm{G}>\mathrm{C}$ locus (rs2488457) in HT patients has three genotypes: $\mathrm{G} / \mathrm{G}, \mathrm{C} / \mathrm{G}$, and $\mathrm{C} / \mathrm{C}$, the frequencies of which are $28 \%, 50 \%$, and $22 \%$, respectively, $\mathrm{G}$ and $\mathrm{C}$ The gene frequencies are $53.8 \%$ and $46.2 \%$, the control group genotype frequencies are $35 \%, 36.7 \%$, and $28.3 \%$, the $\mathrm{G}$ and $\mathrm{C}$ gene frequencies are $51.9 \%$ and $48.1 \%$, respectively. The HT patients and the control group rs 2488457 each genotype and etc. The differences in the distribution frequency of locus genes have statistically significant $(\mathrm{P}<0.05)$, and PTPN22 gene $1123 \mathrm{G}>\mathrm{C}$ locus $\mathrm{C} / \mathrm{G}(\mathrm{OR}=1.727,95 \% \mathrm{CI}$ : $0.203 \sim 1.014), \mathrm{C} / \mathrm{C}$ genotype $(\mathrm{OR}=1.628,95 \% \mathrm{CI}: 0.136 \sim$ 1.283 ) can significantly increase the risk of HT, and carrying $\mathrm{G} / \mathrm{G}$ genotype can reduce the risk of $\mathrm{HT}(\mathrm{OR}=0.722,95 \% \mathrm{CI}$ : $0.128 \sim 0.261)$. This is basically consistent with the results of Pan Ruowang [36] and HuoYunli et al. [37], which suggests that the PTPN gene promoter $1123 \mathrm{G}>\mathrm{C}$ SNP is related to the onset of HT in Chinese population, and the $1123 \mathrm{C}$ gene may be a susceptibility gene for $\mathrm{HT}$, carrying $\mathrm{C} / \mathrm{G}, \mathrm{C} / \mathrm{C}$ genotype may be a risk factor for $\mathrm{HT}$, and carrying $\mathrm{G} / \mathrm{G}$ genotype may be a protective factor for HT.

Iodine excess is positively correlated with thyroid disease. High iodine intake can affect the secretion of thyroid hormones and the level of autoantibodies in HT patients, and accelerate the development of subclinical hypothyroidism to clinical hypothyroidism, which suggests different iodine nutrition status is closely related to the stratification of HT thyroid function and the degree of thyroid function damage caused by antibody titer. Thyroid function, autoantibodies and Th17/Tregs cell ratio and dysfunction, corresponding cytokines and transcription factors in HT patients with different iodine nutritional status have corresponding changes, and they jointly participate in the occurrence and development of HT. However, there are few researches on the correlation between Th17/Treg cytokines and PN22 gene Qigongzi-1123 genotype in HT patients with different iodine nutritional status. The author's research [38] finds that the composition ratio of HT patients with different urinary iodine concentrations, the 
sum of iodine overdose and iodine overdose group is consistent with the proportion of iodine adequate group, which is significantly higher than that of iodine deficiency group. The MUI in the clinical hypothyroidism group of HT patients is negatively correlated with serum FT3, FT4, TT3, and TT4 $(\mathrm{r}=-0.258, \mathrm{P}=0.039 ; \mathrm{r}=-0.296, \mathrm{P}=0.029 ; \mathrm{r}=-0.216$, $\mathrm{P}=0.043 ; \mathrm{r}=-0.208, \mathrm{P}=0.046) ;$ MUI in the subclinical hypothyroidism group is negatively correlated with serum FT3 and FT4 $(\mathrm{r}=-0.289, \mathrm{P}=0.038 ; \mathrm{r}=-0.301, \mathrm{P}=0.0349)$, and positively correlated with $\mathrm{TSH}(\mathrm{r}=0.295, \mathrm{P}=0.028)$. In the iodine deficiency group, the TSH level is higher than the other 3 groups. As the urine iodine level increased, the serum TPO-Ab and TG-Ab levels in the HT 3 group also increase. Further research [39] finds that the changes of Th17 and Tregs cells and their cytokines and transcription factors are basically the same as those of HT patients with iodine deficiency, adequate iodine, iodine excess, and iodine beyond. The changes in HT patients are basically the same as those of HT patients. The decrease of cell ratio and the increase of Th17/Treg ratio and IL-17 are more obvious, but there have no statistically significant differences between the groups. This research finds that there has no statistically significant difference between PTPN22 gene $-1123 \mathrm{G}>\mathrm{C}$ site (rs2488457) $\mathrm{G} / \mathrm{G}, \mathrm{C} / \mathrm{G}, \mathrm{C} / \mathrm{C}$ genotype and $\mathrm{G}$ and $\mathrm{C}$ gene frequencies in HT patients with different iodine nutritional status and the control group. It shows that the iodine nutritional status and the $-1123 \mathrm{G}>\mathrm{C}$ polymorphism of the PTPN22 gene have no correlation with the onset of HT, and may also be related to the small number of test samples, which needs to be further confirmed by a large-sample prospective study. Although the study of iodine nutritional status and PTPN22 gene in the pathogenesis of HT does not obtain positive results, it also opens up a new way for the study of environmental and genetic heterogeneity in the pathogenesis of HT.

At present, it is believed that the function of LYP to inhibit TCR signal transduction of $\mathrm{T}$ cells may be through the following ways: (1) Combining with Csk and synergistically down-regulate signal transduction, dephosphorylate Zap70 and $\mathrm{Src}$ and other protein tyrosine kinase families to make TCR Related kinases are inactivated to limit the degree of T cell response to antigens; (2) By inhibiting the SH3 region of Csk, it participates in the inhibition of the T cell receptor (TCR) signaling system; (3) Through the C-terminal P1 motif and protein tyrosine, The $\mathrm{SH} 3$ domain of the enzyme (PTP) is connected to cooperate with Csk to inhibit T cell activation, thereby playing a negative regulatory role in inhibiting the process of $\mathrm{T}$ cell activation [8]. However, the mechanism by which PTPN22-1123 gene polymorphism affects the function of LYP is still unclear. DNA sequence analysis found that the -1123 site matched the binding site of transcription factor activator protein 4 (AP-4), and it happened to be in the core position of the AP-4 binding consensus sequence (-1130gcaaGCTGaa-1121) [40]. The expression of the wild-type $\mathrm{G}$ allele is down-regulated after the conversion to the $\mathrm{C}$ allele, which affects the transcription regulation function of the PTPN22 promoter, so that the $-1123 \mathrm{C}$ allele cannot match the binding elements of any known transcription factors, which may affect the Lyp Transcription efficiency. However, the specific mechanism of PTPN22-1123 gene polymorphism in the development of HT still needs to be further studied.

In summary, the thyroid function of HT patients with different iodine nutritional status changes to varying degrees, often accompanied by significant increases in TPOAb and $\mathrm{TgAb}$ titers, and there is a certain correlation with Th17/Treg cells and their cytokines and transcription factors. It shows that HT is mainly an immune response mediated by Th17 cells and is accompanied by abnormalities in humoral immunity. PTPN22 gene polymorphism may be related to susceptibility to HT. Carrying $\mathrm{C} / \mathrm{G}$ and $\mathrm{C} / \mathrm{C}$ genotypes may increase the risk of HT and is a risk factor for HT. Detection of PTPN22 gene SNP may help evaluate the risk of HT. It is further confirmed that there is a certain correlation between Th17/Treg cytokines, transcription factors and PTPN22 gene polymorphisms in HT patients, which may be immune and genetic factors that affect the occurrence and development of HT, but different iodine nutritional status and PTPN22 gene polymorphisms has not statistically significant. In-depth research on the interaction and mechanism of action between Th17/Treg cells and their factors and PTPN22 gene polymorphisms in HT patients with different iodine nutritional status will be useful for the diagnosis, treatment and prevention of HT Open up new areas.

\section{Conclusions}

Iodine excess is positively correlated with thyroid disease. The composition ratio of HT patients with different urinary iodine concentrations is the same as the ratio of the iodine excess and iodine beyond groups to the iodine adequate group, which is significantly higher than the iodine deficiency group. In addition, thyroid function, autoantibodies and Th17/Tregs cell ratio and dysfunction, corresponding cytokines and transcription factors of HT patients with different iodine nutritional status have changed accordingly, and jointly participate in the development of HT. With iodine deficiency, adequate iodine, iodine excess, and iodine beyond in HT patients, the changes in Th17 and Tregs cells and their cytokines and transcription factors are basically consistent with the changes in HT, manifested as a decrease in the proportion of Treg cells and the ratio of Th17/Treg. And IL-17 increases significantly, but the differences between the groups have not statistically significant. PTPN22 gene polymorphism may be related to susceptibility to HT. Carrying C/G and C/C genotypes may increase the risk of HT and is a risk factor for HT. Detection of PTPN22 gene SNP may help evaluate the risk of HT. There is no statistically significant difference between PTPN22 gene $-1123 \mathrm{G}>\mathrm{C}$ site (rs2488457) $\mathrm{G} / \mathrm{G}, \mathrm{C} / \mathrm{G}$, $\mathrm{C} / \mathrm{C}$ genotype and $\mathrm{G}$ and $\mathrm{C}$ gene frequencies in $\mathrm{HT}$ patients with different iodine nutrition status and the control group, which indicates iodine nutrition status and PTPN22 gene $-1123 \mathrm{G}>\mathrm{C}$ polymorphism has no correlation with the pathogenesis of HT. It is further confirmed that there is a certain correlation among Th17/Treg cytokines, transcription factors and PTPN22 gene polymorphisms in HT patients, which may be immune and genetic factors that affect the 
development of HT. Although the research on iodine nutritional status and PTPN22 gene in the pathogenesis of HT does not obtain positive results, it also opens up a new way for the study of environmental and genetic factors in HT. Therefore, in-depth research on the interaction and mechanism of action between Th17/Treg cells and their factors in HT patients with different iodine nutritional status and PTPN22 gene polymorphism will provide a scientific basis for the diagnosis, treatment and prevention of HT.

\section{Acknowledgements}

Cangzhou Key R \& D Plan and Guidance Project (Project No: 1833020011).

Natural Science Foundation of Cangzhou Medical College (No. 18Z015).

\section{References}

[1] Tang QL, Xue YM. Hashimoto's thyroiditis related gene research progress [J]. Drug Research, 2013, 10 (15): 42-46.

[2] Thomas Heiberg Brix and Laszlo Hegedus. twin studies as a model for exploring the aetiology of autoimmune thyroid disease [J]. Clinical Endocrinology, 2012, 76: 457-464.

[3] Shi Y, Wang H, Su Z, et al. Differentiation imbalance of Th1/Th17 in peripheral blood mononuclear cells might contribute to pathogenesis of Hashimoto's thyroiditis [J]. Scand J Immunol. 2010, 72 (3): 250-255.

[4] Wang S, Baidoo SE, Liu Y, et al. T cell-derived leptin contributes to increased frequency of $\mathrm{T}$ helper type 17 cells in female patients with Hashimoto's thyroiditis [J]. Clin Exp Immunol. 2013, 171 (1): 63-68.

[5] XieXY, Xi' erNY, Guo T, et al. TheAlterationoftheTh1, Th2, Th17, Treg Cells in the Peripheral Blood in Patients with Grave' s Disease (GD) and Hashimoto'ds Thyroiditis with Hypothyroidism. J Radioimmunology, 2011, 24 (3): 290-293.

[6] Glick AB, WodzinskiA, FuP, et al. Impairment of regulatory T cell function in autoimmune thyroid disease [J]. Thyroid, 2013, 23 (7): 871-878.

[7] Marazuela M, García-López MA, Figueroa-Vega N, et al. Regulatory $\mathrm{T}$ cells in human autoimmunethyroiddisease $[\mathrm{J}]$. Clin Endocrinol Metab. 2006, 91 (9): 3639-3646.

[8] Chen MH, DaiB. Relationship between protein tyrosine phosphatase nonreceptor type 22 and rheumatoid arthritis [J]. Foreign Medicals Ciences Ection of Medgeography, 2014, 35 (3): 252-255.

[9] Li Sheng Dong. Analysis of association between PTPN22 and transcription factor FOXP3 gene polymorphisms and genetic susceptibility to systemic lupus erythematosus [J]. Chin Mode Doct, 2017, 55 (5): 1-4.

[10] $\mathrm{Su}$ Y. Association Study between PTPN22 Gene Polymorphism and Myasthenia Gravis [D]. Master's thesis of shandong university, 2019.

[11] Xu QH, Wu J, Chen ZT. Advances in the Research of the SPNs of PTPN22 Gene and Autoimmune Related Diseaes [J]. Med J Wuhan Unive, 2017, 38 (5): 844-849.
[12] Chinese Medical Association Endocrine Credits "China thyroid disease treatment guidelines" Writing Group. China thyroid disease treatment guidelines-Thyroiditis $[\mathrm{J}]$. Chin $\mathrm{J}$ inter Med, 2008, 47 (9): 784-788.

[13] Chinese Medical Association Endocrine Credits "China thyroid disease treatment guidelines" Writing Group. China thyroid disease treatment guidelines-iodine deficiencydisorders [J]. Journal of Internal Medicine, 2008, 47 (8): 689-690.

[14] Kleinewietfeld M, Hafler DA. The plasticity of human Treg and Th17 cells and its role in autoimmunity [J]. Semin Immunol. 2013, 25 (4): 305-312.

[15] SinghB, SchwartzJA, SandrockC, et al. Modulation of autoimmune diseases by interleukin (IL)-17 producing regulatory T helper (Th17) cells [J]. Indian J Med Res, 2013, 138 (5): 591-594.

[16] Uqba Khan, Ghazanfar H. T Lymphocytes and Autoimmunity [J]. Int Rev Cell Mol Biol. 2018, 341: 125-168.

[17] Chang HH, Tai TS, Lu B, et al. ChristinePTPN22.6, a dominant negative isoform of PTPN22 and potential biomarker of rheumatoid arthritis [J]. PLoS One. 2012, 7 (3): e33067.

[18] Zheng K, Zhang J, Zhang P. et al. PTPN22 and CTLA-4 gene polymorphisms in resected thymomas and thymus for myasthenia gravis [J]. Thorac Cancer. 2012, 3 (4): 307-312.

[19] Liu J, Chen M, Li R, et al. Biochemical and functional studies of lymphoid-specific tyrosine phosphatase (Lyp) variants S201F and R266W [J]. PLoS One. 2012, 7 (8): e43631.

[20] Xuan C, Lun LM, Zhao JX, et al. PTPN22 gene polymorphism (C1858T) is associated with susceptibility to type 1 diabetes: a meta-analysis of 19, 495 cases and 25, 341 controls [J]. Ann Hum Genet, 2013, 77 (3): 191-203.

[21] Lavrikova EIu, Nikitin AG, Seregin IA, et al. Association of the C1858T polymorphism of the PTPN22 gene with type 1 diabetes [J]. MolBiol (Mosk), 2009, 43 (6): 1040-1043.

[22] Wang L, Zhang L, Xiao $\mathrm{H}$, et al. Interference of protein tyrosine phosphatase non-receptor type 22 inhibits NLRP3 inflammasome activation to alleviate inflammation after cerebral hemorrhage in rats [J]. J Third Milit Med Univ, 2019, 41 (24): 2409-2416.

[23] Kawasaki E, Awata T, Ikegami H, et al. Systematic search for single nucleotide polymorphisms in a lymphoid tyrosine phosphatase gene (PTPN22): association between a promoter polymorphism and type 1 diabetes in Asian populations [J]. Am J Med Genet A, 2006, 140 (6): 586-593.

[24] Salama A, Elshazli R. 1said A, et al. rotein tyrosine phosphatase non-receptor type $22($ PTPN22) $+1858 \mathrm{C}>\mathrm{T}$ gene polymorphism in Egyptian cases with rheumatoid arthritis [J]. Cell Immunol, 2014, 290 (1): 62-65.

[25] Zhao L, Qiu YR. Associations of Protein Tyrosine Phosphatase Nonreceptor 22 (PTPN22) Gene Polymorphisms with RA in the Han Population of Guangzhou in China [J]. J Tropical Med, 2009.9 (5): 493-495.

[26] Wang YP, Zhou Y, Fei Y. Relationship between PTPN22 gene polymorphism and rheumatoid and lupuserythematosus [J]. Chin J Public Health, 2009, 25 (12): 1535-1536.

[27] Bai SY, Zhang LM, Wang CX, et al. Study on the relationship between polymorphism of PTPN22 and rheumatoid arthritis [J]. J Taishan Med Colle, 2010, 31 (2): 100-102. 
[28] Kong XT, Wang LH, Wang JJ, et al. Research progress on the correlation between PTPN22 C1858T and autoimmune diseases [J]. Chin J Neuroimmunol \& Neurol, 2016, 23 (6): 442-447.

[29] Hamza RT, Awwad KS, Temsah KA, et al. R620W polymorphism of protein tyrosine phosphatase PTPN22 in Egyptian children and adolescents with systemic lupus erythematosus: relation to thyroid autoimmunity $[\mathrm{J}]$. Int $\mathrm{J}$ Adolesc Med Health. 2013, 25 (2): 143-149.

[30] Tang L, Wang Y, Zheng S, et al. PTPN22 polymorphisms, but not R620W, were associated with the genetic susceptibility of systemic lupus erythematosus and rheumatoid arthritis in a Chinese Han population [J]. Hum Immunol. 2016, 77 (8): 692-698.

[31] Zhen RZ, Li R, Zhang SH. PTPN22 gene polymorphism and autoimmune endocrine diseases [J]. Inte J Endocrinol Metab, 2007, 27 (2): 89-91.

[32] Alkhateeb A, Marzouka NA, TashtoUShR. Variants in PTPN22 and SMOC2 genes and the risk of thyroid disease in the Jordanian Arab population [J]. Endocrine, 2013, 44 (3): 702-709.

[33] Ray D, Tomar N, Gupta N, et al. Protein tyrosine phosphatase non-receptor type 22 (PTPN22) gene R620W variant and sporadic idiopathic hypoparathyroidism in Asian Indians [J]. Int J Immuno-genet. 2006, 33 (4): 237-240.

[34] Ban Y, Tozaki T, Taniyama M, et a1. Association of the protein tyrosine phosphatase nonreceptor 22 haplotypes with autoimmune thyroid disease in the Japanese population [J]. Thyroid, 2010, 20 (8): 893-839.

[35] Xue L, Pan C, Gu Z, et al. Genetic heterogeneity of susceptibility gene in different ethnic populations: refining association study of PTPN22 for Graves'disease in a Chinese Han population [J]. PLoS One, 2013, 8 (12): e84514.

[36] Pan RW, Zhang EY, Mei WJ, et al. Study on the relationship between PTPN22-1123G $>\mathrm{C}$ gene polymorphism and Hashimoto's Thyroiditis in Wenzhou population [J]. Chin J Healt Labo Techn, 2012, 22 (2): 240-241.

[37] Huo YL, Chang YF, Yang JF, et al. Association between PTPN22 Gene Polymorphism and Hashimoto's Thyroiditis in Zhangiiakou Han Population [J]. Chine J Med Guide, 2017, 19 (12): 1306-1309.

[38] Li HY, Ma JQ, Wang CC, et al. Correlation Analysis between Thyroid Function and Autoantibodies in Hashimoto Thyroiditis Patients with Different Iodine Nutritional Status [J]. Amer J Biom Life Scie 2021, 9 (1): 10-19.

[39] Wang CC, Mu ZX, Chen YX, et al. Study on Th17/Treg Cells and Cytokines in Hashimoto's Thyroiditis with Different Iodine Nutrition Status [J]. Science J Public Health, 2021, 9 (1): 12-22.

[40] Juliger S, Bongartz M, Luty AJ, et al. Functional analysis of a promoter variant of the gene encoding the interferon-gamma receptor chainI [J]. Immunogenetics, 2003, 54 (10): 675-680. 